\title{
The mortality of colorectal cancer in relation to the initial symptom at presentation to primary care and to the duration of symptoms: a cohort study using medical records
}

\author{
S Stapley', TJ Peters' ${ }^{2}$ D Sharp ${ }^{2}$ and W Hamilton ${ }^{* 1,2}$ \\ 'CAPER Research Practices, Halford Wing, Dean Clarke House, Exeter, EXI IPQ, UK; ${ }^{2}$ Academic Unit of Primary Health Care, Department of \\ Community Based Medicine, University of Bristol, The Grange, I Woodland Road, Bristol, BS8 IAU, UK
}

\begin{abstract}
The association between the staging of colorectal cancer and mortality is well known. Much less researched is the relationship between the duration of symptoms and outcome, and whether particular initial symptoms carry a different prognosis. We performed a cohort study of 349 patients with primary colorectal cancer in whom all their prediagnostic symptoms and investigation results were known. Survival data for 3-8 years after diagnosis were taken from the cancer registry. Six features were studied: rectal bleeding, abdominal pain, diarrhoea, constipation, weight loss, and anaemia. Two of these were significantly associated with different staging and mortality. Rectal bleeding as an initial symptom was associated with less advanced staging (odds ratio from one Duke's stage to the next $0.50,95 \%$ confidence interval $0.31,0.79 ; P=0.003$ ) and with reduced mortality (Cox's proportional hazard ratio (HR) 0.56 $(0.4 \mathrm{I}, 0.79) ; P=0.00 \mathrm{I}$. Mild anaemia, with a haemoglobin of $10.0-12.9 \mathrm{~g} \mathrm{dl}^{-1}$, was associated with more advanced staging (odds ratio $2.2(1.2,4.3) ; P=0.021)$ and worse mortality $(H R I .5(0.98,2.3): P=0.064)$. When corrected for emergency admission, sex, and the site of the tumour, the HR for mild anaemia was I.7 (I.I, 2.6); $P=0.015$. No relationship was found between the duration of symptoms and staging or mortality.

British Journal of Cancer (2006) 95, I32I-1325. doi: I0.1038/sj.bjc.6603439 www.bjcancer.com
\end{abstract}

Published online 24 October 2006

(C) 2006 Cancer Research UK

Keywords: colorectal cancer; primary health care; diagnosis; mortality

It is generally assumed that making a diagnosis of colorectal cancer as early as possible is beneficial. Earlier diagnosis can arise from screening or improved recognition of symptomatic cancers. In the UK, approximately three-quarters of colorectal cancers present initially to their general practitioner (GP) with nonemergency symptoms, such as rectal bleeding, abdominal pain, or change in bowel habit (Barrett et al, 2006). Even after the proposed introduction of screening in the UK in 2006, it is likely that the majority of patients will still present with symptoms (Hamilton et al, 2005).

Patients who attend primary care in the UK with clinical features suggesting possible colorectal cancer can be referred to a rapid investigation facility, 'the 2-week clinic.' Referral guidance for these clinics outlines clinical scenarios deemed to carry sufficient risk to warrant rapid investigation. (NICE, 2005) The assumption behind these clinics is that cancers have a symptomatic phase of sufficient duration to offer the possibility of earlier diagnosis, leading to mortality benefits. This assumption can be explored by examination of the relationships between mortality (or staging) and the duration of symptoms. Several such studies have been

* Correspondence: Dr W Hamilton; Academic Unit of Primary Health Care, Department of Community Based Medicine, University of Bristol, The Grange, I Woodland Road, Bristol, BS8 IAU, UK

E-mail:w.hamilton@bristol.ac.uk

Received 6 July 2006; revised 20 September 2006; accepted 21 September 2006; published online 24 October 2006 reported. There is a clear relationship between the staging of the cancer and mortality (Mulcahy and O'Donoghue, 1997; Roncoroni et al, 1999; Ponz de Leon et al, 2000; Gonzalez-Hermoso et al, 2004; Olsson et al, 2004). Conversely, most studies have shown no relationship between the duration of symptoms and staging or mortality (Stubbs and Long, 1986; Barillari et al, 1989; Kyle et al, 1991; Mulcahy and O’Donoghue, 1997; Majumdar et al, 1999; Roncoroni et al, 1999; Kiran and Glass, 2002; Gonzalez-Hermoso et al, 2004; Olsson et al, 2004; Bharucha et al, 2005; Khattak et al, 2006; Rupassara et al, 2006). Some have reported an inverse relationship, with a shorter duration of symptoms being associated with both worse staging and worse prognosis (Mulcahy and O'Donoghue, 1997; Olsson et al, 2004; Rupassara et al, 2006). Once emergency admissions, which generally have a shorter duration of symptoms, are corrected for, this inverse relationship disappears (Mulcahy and O'Donoghue, 1997; Olsson et al, 2004; Rupassara et al, 2006). Only two previous studies have examined the relationship between the first symptom of the cancer and mortality (Gonzalez-Hermoso et al, 2004; Korsgaard et al, 2006). However, all these previous studies have either dated the onset of symptoms from the dates given in the doctor's referral letter, or by interviewing patients after the diagnosis had been made. These methods are prone to inaccuracy, with some patients able only to specify the calendar year that their symptoms began, and others giving illogical dates of onset, such as dates after their diagnosis had been made (Olsson et al, 2004). Furthermore, only two studies were community based, including all cases from a specific area (Olsson et al, 2004; Korsgaard et al, 2006). The 
remainder were hospital-based, raising the possibility that cases referred to that hospital were not typical of the whole colorectal cancer population.

We sought to address these methodological issues by an analysis of a cohort of 349 patients with colorectal cancers occurring in a 5-year period from Exeter, Devon, UK (Hamilton et al, 2005). In this cohort, all symptoms recorded in primary care before diagnosis were collected and coded systematically. This methodology eliminates concerns about the accuracy of patient recall, although it does not address symptoms experienced but not reported to primary care.

\section{MATERIALS AND METHODS}

\section{Participants}

We studied subjects from a previously described population-based case-control study (Hamilton et al, 2005). In that study, all 358 primary colorectal cancer cases in patients aged 40 years or more from Exeter Primary Care Trust (PCT), diagnosed between 1998 and 2002, were identified from the local cancer registry. This list was augmented by computer searches at all 21 general practices in the PCT, identifying a further three cases. Twelve of the total 361 cases could not be studied as they had either died (five) or left Exeter (seven), and their primary care records were unobtainable. Therefore, 349 cases with full primary care records were studied, $141(40 \%)$ of whom had also died by the time of the initial study, but whose records were retrievable. Emergency presentations were defined as those requiring surgical admission for suspected bowel obstruction or perforation, and who had their cancer diagnosed during the admission, almost always at laparotomy. The entire primary care record for the 2 years preceding diagnosis for all subjects was coded using the International Classification of Primary Care-2, (WONCA, 1998) although for this paper only data from the year before diagnosis were used. The second year before diagnosis was omitted because the rate of reporting of the various clinical features was similar in both cases and controls for that year, so reporting of features in that year by cases would probably be unrelated to their future cancer (Hamilton et al, 2005).

Survival data were obtained from the cancer registry, up to the 8 December 2005, so were available for between 3 and 8 years. Deaths are routinely notified to the registry. Almost all cancerrelated therapy for patients in Exeter PCT takes place at the Royal Devon and Exeter Hospital: attendances to this hospital are also reported to the cancer registry. The last hospital attendance was taken as the last date in which the patient was known to be alive. In 13 patients who had no hospital attendances recorded, nor had been reported dead, their vital status was ascertained from the Patient and Practitioner Services Agency: one patient's details could not be found there, and her status was obtained by direct enquiry at her general practice.

\section{Clinical features studied}

Ten clinical features had been identified as being independently associated with the diagnosis of colorectal cancer: rectal bleeding, abdominal pain, diarrhoea, constipation, weight loss, abdominal tenderness, anaemia (haemoglobin below $13 \mathrm{~g} \mathrm{dl}^{-1}$ ), abnormal rectal examination, a blood sugar level over $10 \mathrm{mml}^{-1}$, and a positive faecal occult blood testing (Hamilton et al, 2005). For this analysis, only the five symptoms and one investigation, anaemia, were included. For simplicity, these are called features from now on, with the index feature being the first one present. Three of the other four features: abdominal tenderness, a rectal mass, and a positive faecal occult blood test, would generally be expected to be identified only in patients who had described a symptom. It was therefore more appropriate to study the symptom itself. The final feature associated with colorectal cancer was a raised blood sugar. Clinically, this finding would be of little practical value in identifying symptomatic colorectal cancer, so it was not studied here. Furthermore, the six selected features mirror those used in previous studies, allowing comparisons to be made.

\section{Analyses}

Two research questions were investigated. The first was that the staging and/or mortality of patients would differ depending on the index feature the patient had experienced in the year before diagnosis. Differences in staging by index feature were analysed using ordinal regression. Initially, this was a univariable analysis, which was followed by adding age and sex and emergency admission to the models. In a small number of patients the Duke's staging was unknown. The cancer registry considered that the commonest cause of absent staging was most likely to be because the patient had disseminated disease, with laparotomy and formal staging not having been undertaken. Therefore, a second analysis was conducted, adding the patients whose staging was unknown to the Dukes' D group. Mortality by index feature was analysed using Cox's proportional hazards regression model. Following a univariable analysis, multivariable Cox regression added the possible confounders of sex, tumour site (to the right of the splenic flexure, or from the splenic flexure to the rectum), and emergency admission. If multiple features were present at the index consultation, the patient was included under each one.

The second issue was that staging and/or mortality would be related to the duration of symptoms, duration being defined as the interval from presentation of the index feature to diagnosis. This was analysed by dividing the duration into approximate quartiles: $1-30,31-90,91-180$, and 181-365 days before diagnosis. Cox's proportional hazards regression was used to analyse the relationship between duration of symptoms and mortality. The above analyses were then repeated with age, staging, and emergency admissions added to the models. Again, patients with absent staging were added to the Duke's D group and the analyses repeated.

\section{RESULTS}

The cohort contained 349 patients with colorectal cancer. Details of their clinical features, their staging, and whether they had an emergency presentation are shown in Table 1. Age and sex distributions were similar between elective and emergency presentations: $P=0.65$ (Wilcoxon test) and $0.21\left(\chi^{2}\right)$, respectively. There was also no relationship between age and staging: $P=0.35$ (median test). This remained so even when the unknown staging cases were added to Duke's D group. Similarly, there was no relationship between sex and staging: $P=0.91\left(\chi^{2}\right)$ again without a material difference when the unknown staging cases were added to the Duke's D group. Three hundred and nineteen of the 349 cases had reported one of the features: of these 319 , a single feature was noted at the index consultation in $255(73 \%)$, two features in 50 (14\%), and three or more in $14(4 \%)$. In the 319 cases with one of the features, the median time this feature was noted was 97 days before diagnosis (interquartile range (IQR) 44, 218).

\section{Initial symptoms and staging}

The Duke's staging in relation to the initial symptoms is shown in Table 2. The ordinal regressions suggested two initial features were associated with different staging. The first was rectal bleeding with an odds ratio of 0.50 (95\% confidence interval $0.31,0.79)$, $P=0.003$, meaning that rectal bleeding was associated with earlier staging. The second feature was mild anaemia with an odds ratio of $2.2(1.2,4.3), P=0.021$, meaning mild anaemia was associated with later staging. These results were almost unchanged if the patients 
Table I Details of the age, sex, emergency presentation, and clinical features of the cohort

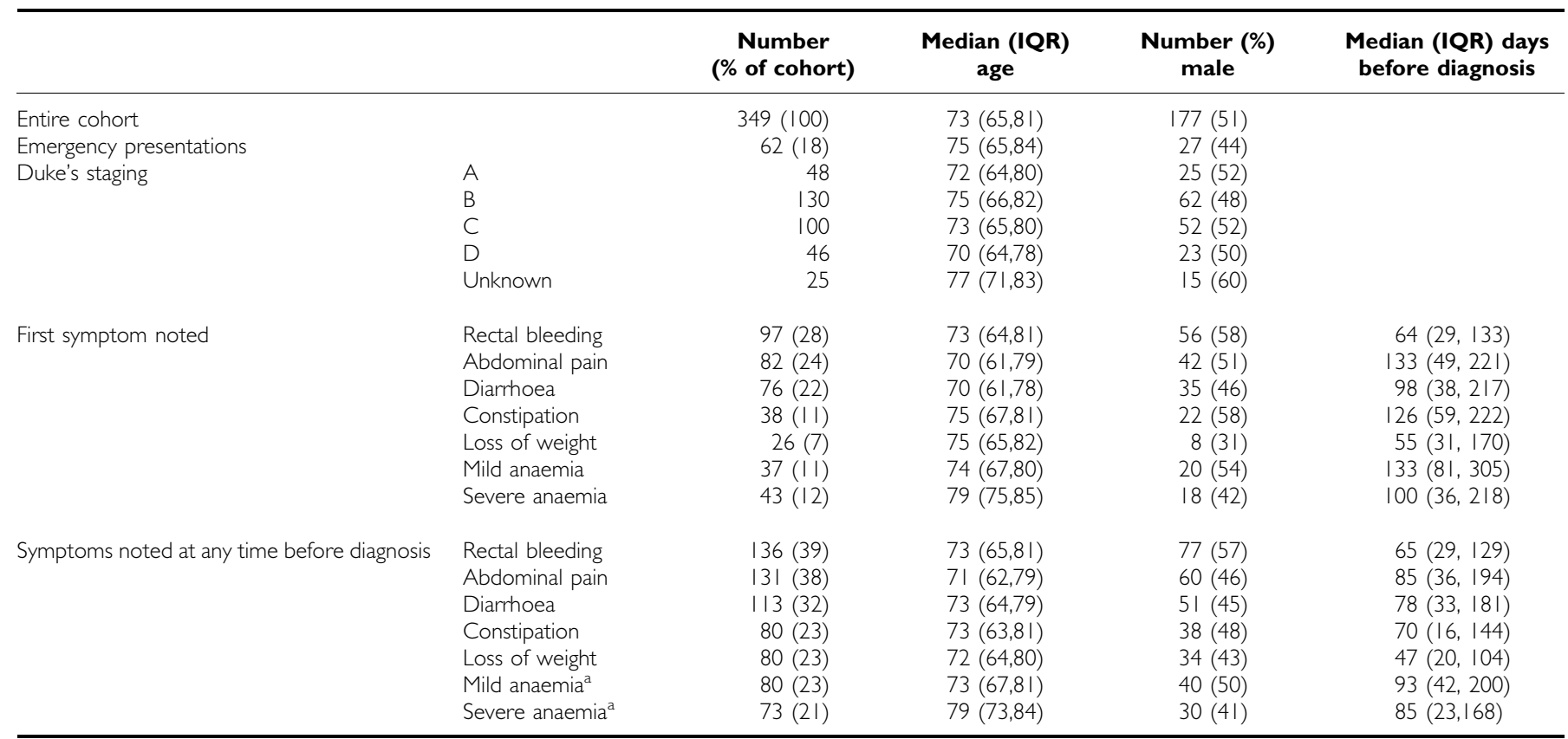

$\mathrm{IQR}=$ interquartile range. ${ }^{\mathrm{a} T}$ wenty-three of these patients had both mild and severe anaemia at some point before diagnosis. Mild anaemia defined as a haemoglobin $10.0-$ $12.9 \mathrm{~g} \mathrm{dl}^{-1}$, severe as $=<9.9 \mathrm{~g} \mathrm{dl}^{-1}$.

Table 2 Duke's staging in relation to the first symptom

\begin{tabular}{lccccccc}
\hline & \multicolumn{6}{c}{ Number (\%) } & \multicolumn{3}{c}{ with each Duke's staging } & \\
\cline { 2 - 6 } First symptom & A & B & C & D & Not known & P-value* \\
\hline Rectal bleeding & $8(8)$ & $24(25)$ & $31(32)$ & $27(28)$ & $7(7)$ & 0.003 \\
Abdominal pain & $6(7)$ & $7(9)$ & $35(43)$ & $23(28)$ & $11(13)$ & 0.59 \\
Diarrhoea & $4(5)$ & $7(9)$ & $32(42)$ & $22(29)$ & $11(14)$ & 0.50 \\
Constipation & $2(5)$ & $5(13)$ & $17(45)$ & $9(24)$ & $5(13)$ & 0.64 \\
Loss of weight & 0 & $2(8)$ & $14(54)$ & $6(23)$ & $4(15)$ & 0.98 \\
Mild anaemia & $3(8)$ & $3(8)$ & $11(30)$ & $10(27)$ & $10(27)$ & 0.021 \\
Severe anaemia & $4(9)$ & $3(7)$ & $18(42)$ & $14(33)$ & $4(9)$ & 0.73 \\
\hline
\end{tabular}

*By ordinal regression.

with unknown staging were added to the Duke's D group, or when age, sex, or emergency admission status were added to the models.

\section{Initial symptoms and mortality}

Of the 349 cases, 207 (59\%) had died by the date of this study. The deaths had occurred a median 421 (IQR 102, 841) days after diagnosis. Follow-up data were available in survivors for a median $1592(1193,2001)$ days after diagnosis. There was no relationship between the number of symptoms recorded at the index consultation and mortality (Table 3 ).

\section{Staging and mortality in relation to the duration of symptoms}

Of the 319 patients with at least one feature of cancer present in the notes, the first feature was recorded between 30 days and 1 day before diagnosis in 61 patients (17\% of the cohort), between 31 and 90 days in 92 patients (26\%), between 91 and 180 days in 62 patients (18\%) and between 181 and 365 days in 104 (30\%) (Table 4).
Table 3 Mortality in relation to the first symptom

\begin{tabular}{lcccc}
\hline & \multicolumn{2}{c}{ Univariable } & \multicolumn{2}{c}{ Multivariable $^{\mathbf{a}}$} \\
\cline { 2 - 5 } First symptom & $\begin{array}{l}\text { Proportional } \\
\text { hazard (CI) }\end{array}$ & P-value & $\begin{array}{c}\text { Proportional } \\
\text { hazard (CI) }\end{array}$ & P-value \\
\hline Rectal bleeding & $0.56(0.41,0.79)$ & 0.001 & $0.57(0.41,0.81)$ & 0.001 \\
Abdominal pain & $1.3(0.93,1.7)$ & 0.14 & $1.3(0.95,1.8)$ & 0.096 \\
Diarrhoea & $0.93(0.67,1.3)$ & 0.70 & $1.1(0.77,1.5)$ & 0.63 \\
Constipation & $1.3(0.84,1.9)$ & 0.27 & $1.1(0.79,1.8)$ & 0.60 \\
Loss of weight & $1.5(0.94,2.4)$ & 0.092 & $1.5(0.91,2.3)$ & 0.11 \\
Mild anaemia & $1.5(0.98,2.3)$ & 0.064 & $1.8(1.1,2.7)$ & 0.010 \\
Severe anaemia & $0.98(0.65,1.5)$ & 0.93 & $0.79(0.51,1.2)$ & 0.30 \\
\hline
\end{tabular}

$\mathrm{Cl}$, confidence interval. ${ }^{a}$ Other variables in model: age, emergency admission status, sex, and site of tumour.

There was no apparent relationship between the duration of symptom in quartiles and staging: $P=0.27\left(\chi^{2}\right.$ test, 12 degrees of freedom (d.f.)). This finding was unchanged if those with unknown staging were added to the Duke's D group; $P=0.67$ ( $\chi^{2}$ test, 9 d.f.).

Mortality in relation to symptom duration is shown in Figure 1. There was no relationship between the duration of symptoms and mortality: $P=0.47$ (Cox proportional hazards). In a multivariable model including symptom duration, there was a relationship between mortality and emergency admission (hazard ratio (HR) 1.9: $P<0.001$ ), age (HR 1.03 for each year older: $P<0.001$ ), and staging (HR 1.5 for each increase in stage: $P<0.001$ ). In this model, left-sided tumours had a survival advantage of marginal significance (HR 0.75: $P=0.059$ ) but again symptom duration was not associated with mortality (HR 1.0: $P=0.11$ ).

\section{DISCUSSION}

The main findings from this study are that the mortality from colorectal cancer differs depending upon the nature of the first 
choose to investigate (du Toit et al, 2006), even though current guidance suggests 6 weeks of isolated bleeding is required to warrant urgent investigation (NICE, 2005). Although severe anaemia is recognised as a feature of possible colorectal cancer, with a risk of approximately 7\% (Logan et al, 2002; NICE, 2005), it is still under-investigated in around half of primary care patients. Mild anaemia, with haemoglobin values in the range $10.0-$ $12.9 \mathrm{~g} \mathrm{dl}^{-1}$, is common in the elderly population, but carries a risk of colorectal cancer of around 1\% (Hamilton et al, 2005). Many GPs would not even consider the possibility of cancer with such mild anaemia - and others may consider investigation of the gastrointestinal tract inappropriate when the risk is relatively low. Nonetheless, it is likely that our finding of increased mortality from colorectal cancer when anaemia is the initial feature will be perpetuated unless doctors change their investigatory behaviour. Testing the stool of such patients for occult blood would be good clinical practice.

This study also suggests that for most patients with a colorectal cancer there is a sufficient interval after symptoms are reported to the doctor for a worthwhile improvement in the timeliness of diagnosis to be possible. Such an improvement would require doctors to identify that their patient may have cancer, and to request appropriate investigation. This may be particularly pertinent when the patient has mild anaemia. Even though the duration of symptoms is unrelated to the outcome of the cancer, it is impossible to construct an argument in favour of delayed diagnosis.

\section{ACKNOWLEDGEMENTS}

This Project was funded by the Department of Health. The funding source had no role in study design, data collection, analysis or writing of the report. All authors had full access to all data, and take final responsibility for publication. WH was funded through his research practice (Barnfield Hill, Exeter) and NHS Fellowships. The views expressed in the publication are those of the authors and not necessarily those of the Department of Health. We wish to thank all 21 general practices in Exeter, the Dendrite personnel, and the Patients and Practitioners Service Agency, without which this project would not have been successful. The three anonymous journal referees also made very sage points, and have improved the paper.

\section{REFERENCES}

Barillari P, de Angelis R, Valabrega S, Indinnimeo M, Gozzo P, Ramacciato G, Fegiz G (1989) Relationship of symptom duration and survival in patients with colorectal carcinoma. Eur J Surg Oncol 15: $441-445$

Barrett J, Jiwa M, Rose P, Hamilton W (2006) Pathways to the diagnosis of colorectal cancer: an observational study in three UK cities. Fam Pract 23: $15-19$

Bharucha S, Hughes S, Kenyon V, Anderson ID, Carlson GL, Scott NA (2005) Targets and elective colorectal cancer: outcome and symptom delay at surgical resection. Colorectal Dis 7: 169-171

du Toit J, Hamilton W, Barraclough K (2006) Risk in primary care of colorectal cancer from new onset rectal bleeding: 10 year prospective study. BMJ 333: 69-70

Gonzalez-Hermoso F, Perez-Palma J, Marchena-Gomez J, Lorenzo-Rocha $\mathrm{N}$, Medina-Arana V (2004) Can early diagnosis of symptomatic colorectal cancer improve the prognosis? World J Surg 28: 716-720

Hamilton W, Round A, Sharp D, Peters T (2005) Clinical features of colorectal cancer before diagnosis: a population-based case-control study. British Journal of Cancer 93: 399-405

Jones R, Rubin G, Hungin P (2001) Is the two week rule for cancer referrals working? BMJ 322: 1555-1556

Khattak I, Eardley NJ, Rooney PS (2006) Colorectal cancer - a prospective evaluation of symptom duration and GP referral patterns in an inner city teaching hospital. Colorectal Dis 8: 518-521

Kiran P, Glass R (2002) Duration of symptoms and spread of colorectal cancer: a short history does not mean early disease. Ann Roy Coll Surg Engl 84: $381-385$

Korsgaard M, Pedersen L, Sorensen HT, Laurberg S (2006) Reported symptoms, diagnostic delay and stage of colorectal cancer: a populationbased study in Denmark. Colorectal Dis 8: 688-695

Kyle SM, Isbister WH, Yeong ML (1991) Presentation, duration of symptoms and staging of colorectal carcinoma. Aust NZ J Surg 61: $137-140$

Logan E, Yates J, Stewart R, Fielding K, Kendrick D (2002) Investigation and management of iron deficiency anaemia in general practice: a cluster randomised controlled trial of a simple management prompt. Postgrad Med J 78: 533 - 537

Majumdar SR, Fletcher RH, Evans AT (1999) How does colorectal cancer present? Symptoms, duration, and clues to location. Am J Gastroenterol 94: $3039-3045$

Mulcahy HE, O'Donoghue DP (1997) Duration of colorectal cancer symptoms and survival: the effect of confounding clinical and pathological variables. Eur J Cancer 33: 1461 - 1467

NICE (2005) Referral Guidelines for Suspected Cancer. London: NICE

Olsson L, Bergkvist L, Ekbom A (2004) Symptom duration versus survival in non-emergency colorectal cancer. Scand J Gastroenterol 39: 252 - 258

Ponz de Leon M, Benatti P, Di Gregorio C, Fante R, Rossi G, Losi L, Pedroni M, Percesepe A, Roncucci L (2000) Staging and survival of colorectal cancer: are we making progress? The 14-year experience of a Specialized cancer Registry. Dig Liver Dis 32: 312-317

Roncoroni L, Pietra N, Violi V, Sarli L, Choua O, Peracchia A (1999) Delay in the diagnosis and outcome of colorectal cancer: a prospective study. Eur J Surg Oncol 25: $173-178$

Rupassara KS, Ponnusamy S, Withanage N, Milewski PJ (2006) A paradox explained? Patients with delayed diagnosis of symptomatic colorectal cancer have good prognosis. Colorectal Dis 8: 423-429

Stubbs RS, Long MG (1986) Symptom duration and pathologic staging of colorectal cancer. Eur J Surg Oncol 12: $127-130$

WONCA (1998) ICPC-2. International Classification of Primary Care. Prepared by the Classification Committee of WONCA. Oxford: Oxford University Press 